\title{
Long-lasting $D$-region ionospheric modifications, caused by intense lightning in association with elve and sprite pairs
}

\author{
Christos Haldoupis, ${ }^{1}$ Morris Cohen, ${ }^{2}$ Benjamin Cotts, ${ }^{3}$ Enrico Arnone, ${ }^{4}$ and Umran Inan ${ }^{2,5}$
}

Received 23 June 2012; revised 17 July 2012; accepted 17 July 2012; published 28 August 2012.

[1] Observations show that intense +CG lightning discharges which trigger both an elve and a sprite are associated with long-lasting conductivity modifications in the upper $D$-region ionosphere. They are observed as strong perturbations in VLF signals propagating through the disturbed region, manifested as LOng Recovery Early VLF events (LORE), which can last up to 30 minutes. These same ionospheric modifications are also responsible for step-like changes, seen mostly in off-storm VLF transmissions, which offset signal levels even for longer times. The evidence suggests that when a very intense positive cloud to ground lightning stroke leads to an elve and a high altitude sprite, and possibly a sprite halo as well, there is production of long lasting elevations in electron density at VLF reflection heights that cause LOREs and severe effects on VLF propagation. The present results confirm past predictions and postulations that elves may be accompanied by long-lasting electron density perturbations in the lower ionosphere. Citation: Haldoupis, C., M. Cohen, B. Cotts, E. Arnone, and U. Inan (2012), Long-lasting $D$-region ionospheric modifications, caused by intense lightning in association with elve and sprite pairs, Geophys. Res. Lett., 39, L16801, doi:10.1029/2012GL052765.

\section{Introduction}

[2] Tropospheric lightning may couple electrical energy, through quasi-electrostatic (QE) and/or electromagnetic (EM) fields, directly into the upper atmosphere and lower ionosphere. This energy coupling is best manifested by the occurrence of "transient luminous events" (TLEs), which are momentary luminous structures of various types, such as "sprites", "sprite halos", "elves", "blue jets", and "gigantic jets" [e.g., see Cummer and Lyons, 2005; Barrington-Leigh et al., 2001; Fukunishi et al., 1996; Pasko et al., 2002]. Lightning discharges may also cause heating and ionization changes directly in the overlying $D$-region ionosphere, therefore leading to conductivity enhancements [Taranenko et al., 1993]. These conductivity enhancements may affect propagation of very low frequency (VLF) transmissions that travel long distances in the Earth-ionosphere waveguide, causing abrupt perturbations in the VLF signal amplitude

\footnotetext{
${ }^{1}$ Department of Physics, University of Crete, Heraklion, Greece.

${ }^{2}$ Department of Electrical Engineering, Stanford University, Stanford, California, USA.

${ }^{3}$ Electrical Engineering and Computer Science Practice, Exponent, Inc., Bowie, Maryland, USA.

${ }^{4}$ Istituto di Scienze dell'Atmosfera e del Clima, CNR, Bologna, Italy.

${ }^{5}$ Koç University, Istanbul, Turkey.

Corresponding author: C. Haldoupis, Department of Physics, University of Crete, GR-71003 Heraklion, Greece. (chald@physics.uoc.gr)

(C2012. American Geophysical Union. All Rights Reserved. 0094-8276/12/2012GL052765
}

and/or phase. These VLF perturbations are known as "early" VLF events and have been studied extensively (see review by Inan et al. [2010]). Optical and VLF correlative studies established a close relation between sprites, sprite halos and occasionally elves, with early VLF perturbations [Haldoupis et al., 2004; Marshall et al., 2006; Mika and Haldoupis, 2008; Haldoupis et al., 2010].

[3] An important and often overlooked property of the early events is their recovery time, ranging typically between 10 and 100 seconds [Inan et al., 2010]. It is controlled by the electron density relaxation time at mesospheric altitudes mostly between 65 and $80 \mathrm{~km}$ [e.g., Pasko et al., 1995; Moore et al., 2003; Haldoupis et al., 2009]. In addition to the common early events, Cotts and Inan [2007] reported a much less frequent class, characterized by much longer recoveries of up to 30 minutes. The same study also reported step-like early-events, in which the observed perturbations do not recover to pre-onset values for at least 30 minutes to an hour. Cotts and Inan [2007] relied on theoretical calculations by Lehtinen and Inan [2007] to postulate that the long recovery events result from lower-altitude $(<50 \mathrm{~km})$, long-lasting conductivity relaxation times associated with the mutual neutralization of long-lived heavy ions in the lower ionosphere. They went further to suggest an association with "Gigantic Jets" (GJs), which are huge upward discharges connecting the top of a thundercloud at $\sim 10 \mathrm{~km}$ with the upper $D$-region ionosphere near $90 \mathrm{~km}$ [Pasko et al., 2002].

[4] In this letter we present key observational evidence showing that early VLF events with long-lasting and steplike recoveries occur in association with simultaneous large elves and high altitude sprites, both triggered together by very intense positive cloud to ground (+CG) lightning discharges. We propose that LOREs (LOng Recovery Early VLF events) are caused by large and long-lived electron density enhancements in the uppermost $D$-region ionosphere.

\section{Experiments and Data}

[5] The present observations include lightning, TLE video images, and VLF radio recordings during a localized thunderstorm that occurred over the sea about $100 \mathrm{~km}$ west of Corsica, around $42^{\circ} \mathrm{N} ; 7.5^{\circ} \mathrm{E}$. It lasted for $\sim 6$ hours during the night of 12-13 December 2009, from about 21-03 UT, and produced many TLE events. After 22:20 UT, when video observations started, there were a total of 50 TLEs recorded, mostly sprites; among them were 8 sprite-elve pairs and a few sprite halos. There was also a spectacular gigantic jet, the first observed in Europe, which was studied in detail by van der Velde et al. [2010].

[6] The TLE observations were taken from the Italian mainland by Ferruccio Zanotti, a member of the Italian 


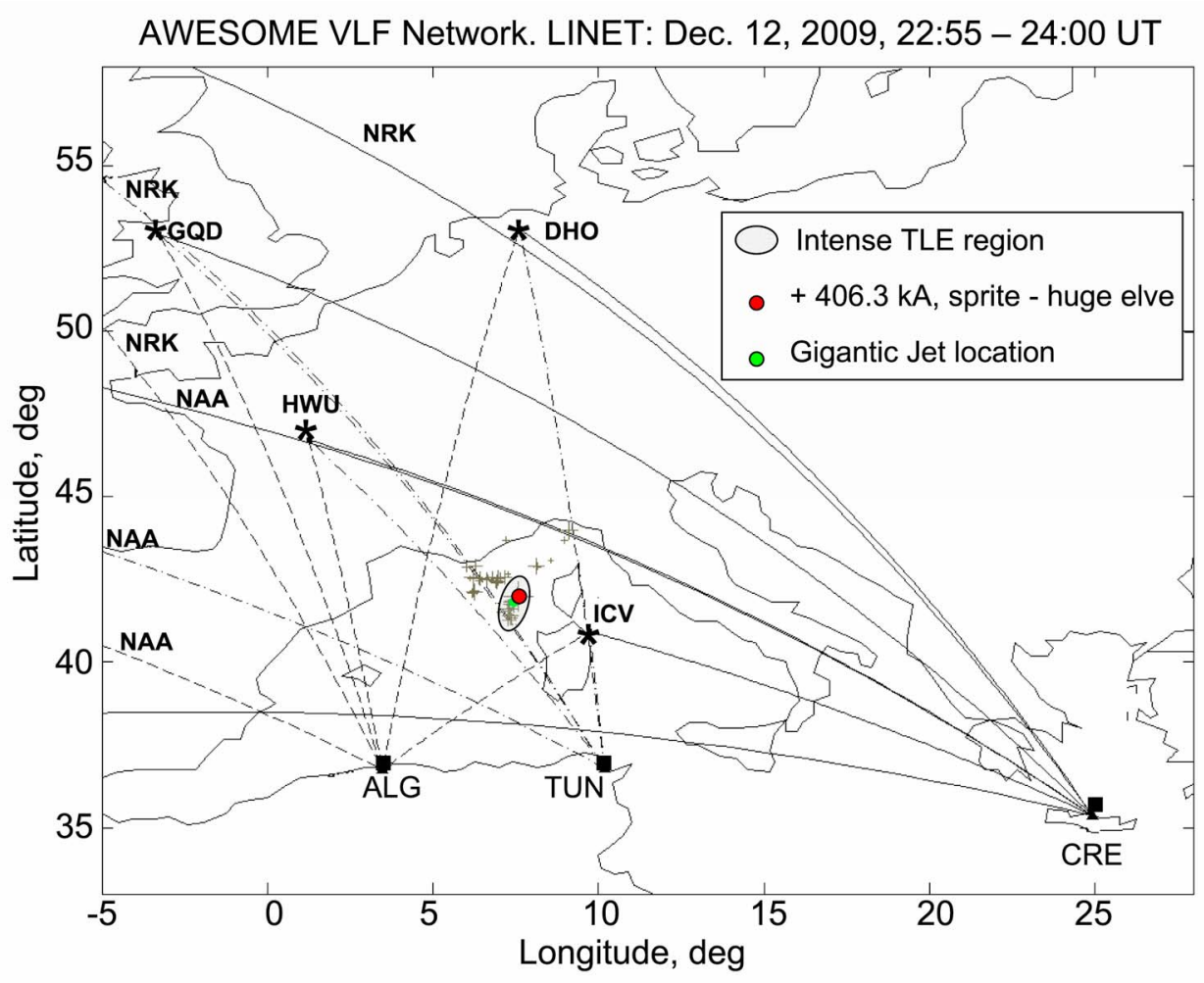

Figure 1. Geographic map showing transmitter (asterisk) and receiver (square) locations and great circle paths (GCPs) of the AWESOME VLF network in Europe and North Africa. The storm that produced the TLEs used in this study was located over the Mediterranean sea $\sim 100 \mathrm{~km}$ west of Corsica. See text for details.

Meteor and TLE network (http:/www.imtn.it/), using a portable CCD color video camera located in Montignoso $\left(44.01^{\circ} \mathrm{N}\right.$ and $\left.10.15^{\circ} \mathrm{E}\right)$, about $300 \mathrm{~km}$ northeast of the storm. The lightning data were provided by the European LIghtning detection NETwork (LINET). The VLF recordings were obtained with 3 narrowband receivers $(\mathrm{Rx})$ located in Tunis, Crete and Algiers, all of which are part of the Stanford University AWESOME international VLF network [Scherrer et al., 2008]. The AWESOME receivers [see Cohen et al., 2010], are identical units using accurate GPS timing to sample at $20 \mathrm{~ms}$ the signals of various VLF transmitters (Tx) located around the globe.

[7] Figure 1 shows a map of VLF receiver locations (squares) and transmitters (asterisks), and great circle paths (GCPs) used in this study. We use four European transmitters (GQD-22.1 kHz, UK; DHO-23.4 kHz, Germany; HWU$20.9 \mathrm{kHz}$, France; ICV-20.27 kHz, Italy), along with NAA$24.0 \mathrm{kHz}$ in Cutler ME, USA, and NRK- $37.5 \mathrm{kHz}$, in Keflavik, Island. Figure 1 also shows crosses identifying $+\mathrm{CG}$ lightning discharges measured by LINET from 22:55 to 24:00 UT. The shaded area depicts the region that produced powerful $+\mathrm{CG}$ discharges responsible for the strongest TLEs that occurred in relation with LOREs. Out of the 18 VLF links used in the present study, the NRK, GQD and HWU links to Tunis are the best suited for observing early VLF perturbations because their Tx-Rx GCPs pass through or near $(<100 \mathrm{~km})$ the storm, a condition that is necessary for narrow angle (forward) scattering and early VLF event detection [Poulsen et al., 1993; Johnson et al., 1999; Haldoupis et al., 2010]. The rest of the Tx-Rx links, depicted also in Figure 1, have GCPs which are not optimal for early event detection because they pass relatively far from the storm, at distances larger than $\sim 200$ to $300 \mathrm{~km}$.

\section{Observations}

[8] The present letter discusses LOREs and their association to TLEs. There were at least 10 LOREs which occurred during the storm, with recoveries ranging from 5-30 min. Analysis of all available data reveals that the stronger events are characterized by unusually large amplitude $(5-10 \mathrm{~dB})$ and phase perturbations $\left(20^{\circ}-80^{\circ}\right)$ which occurred mostly in association with intense $+\mathrm{CG}$ lightning discharges (mostly from 150-400 kA peak currents) that produced both sprites and elves in the upper $D$-region ionosphere.

[9] The most prominent event was triggered by a powerful +CG 406.3 kA lightning stroke at 23:41:07.2613 UT, which was the strongest measured by LINET during the entire storm [van der Velde et al., 2010]. This huge lightning discharge produced a spectacular elve at higher $D$-region altitudes as well as a cluster of sprite carrots and likely a sprite halo as well. The elve emissions expanded out radially over large distances, forming a momentary ring of light with an outer diameter estimated at $\sim 500 \mathrm{~km}$. This exceptional situation was accompanied by the onset of a long lasting modification of the upper $D$-region ionosphere, evidenced by a pronounced LORE in both VLF amplitude and phase. The onset amplitude perturbations of the "near-storm" HWU and NRK links to Tunis reached 8 and $5 \mathrm{~dB}$, respectively, while it took them both more than 25 minutes to return back to pre-event levels. In addition this event also caused step 


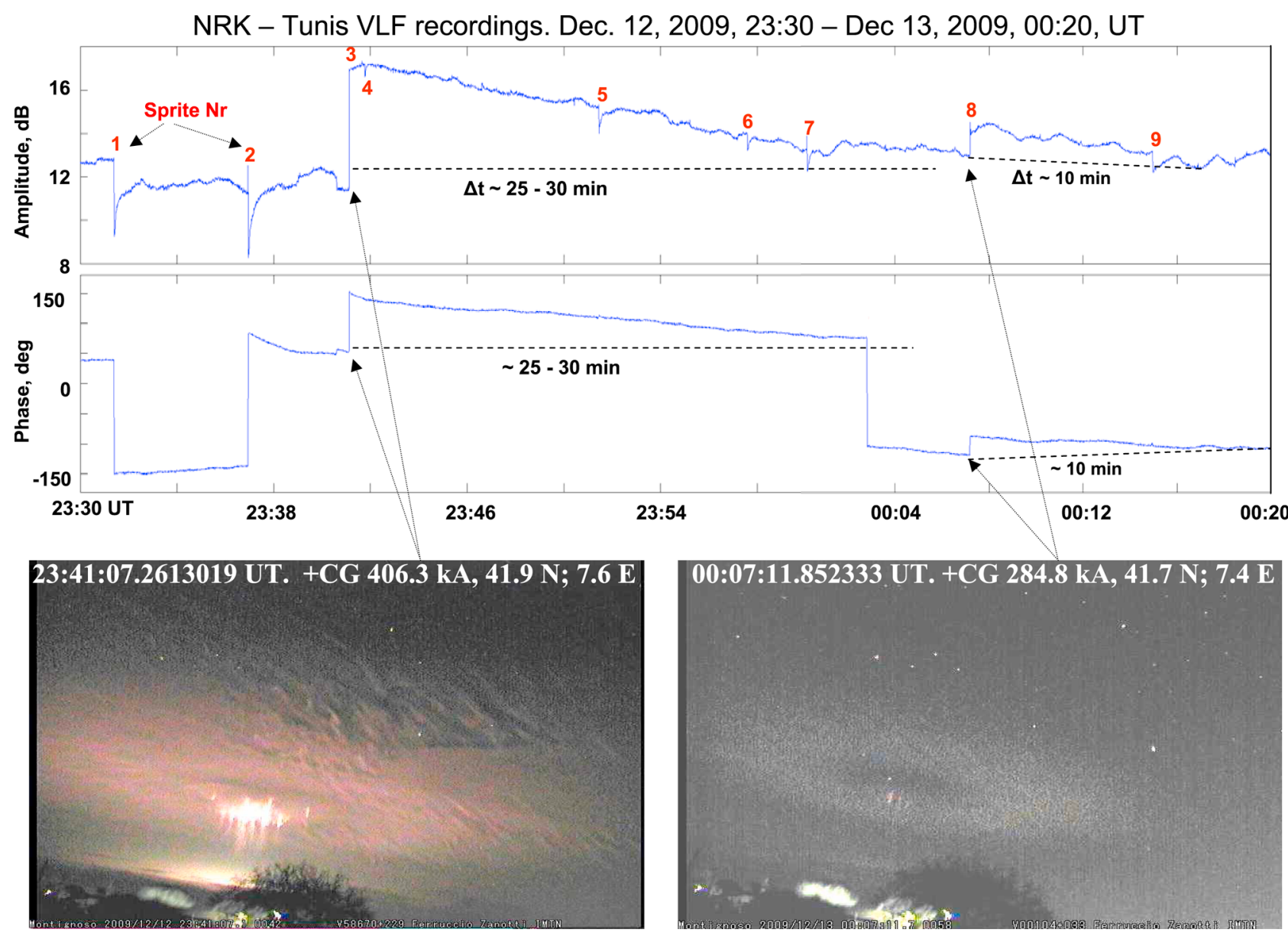

Figure 2. Large amplitude and phase perturbations of long recovery early VLF events caused by very intense $+C G$ discharges, which trigger large elves and sprites in the upper $D$-region and apparently also long-lasting electron density perturbations.

like early perturbations in several VLF transmissions received in Algiers and Crete, for which their GCPs were "far-storm", passing at relatively large distances from the causative lightning flash. Surprisingly, the event caused no perturbation in the GQD-Tunis link despite that its GCP cut through the storm area (Figure 1).

[10] Figure 2 documents the main characteristics of the event. Shown in the upper part are time series of signal amplitude and phase for the NRK-Tunis link, recorded during the storm interval 22:30 to 00:20 UT of Dec. 12-13, 2009. During this time, there were 9 sprites observed (marked in Figure 2 by a count number) which occurred in full correspondence with onsets of early VLF events, in line with what has been reported by Haldoupis et al. [2004, 2010]. As seen in the lower left panel image, the exceptional + CG discharge triggered both an upper altitude sprite and an elve. The latter was immense, extending over most of the camera's $56^{\circ}$ field of view and momentarily illuminating a large area of the lower thermosphere. Actually, the elve acted as a snapshot, revealing wave-like formations in neutral density that display quasi-parallel tilted striations, most likely caused by the downward phase-propagation of atmospheric gravity waves.

[11] The amplitude and phase recoveries of the LORE in Figure 2 persist for $\sim 25$ min until a subsequent $+\mathrm{CG}$ $284.8 \mathrm{kA}$ lightning flash at 00:07:11.8523 UT triggered a smaller signal perturbation. As shown in the lower-right image of Figure 2, this same lightning flash also produced a few very faint sprite columns and a large elve ring. The signal perturbation here is also a LORE, with an amplitude and phase recovery lasting for $\sim 10 \mathrm{~min}$. The rest of the early events in Figure 2 were typical, having recoveries $<100$ seconds. To the time resolution of the CCD camera these were associated only with usual mesospheric sprites, in line with what is well known from previous studies [e.g., see Haldoupis et al., 2010].

[12] In addition to the significantly longer than typical recovery times of LOREs, the LORE recovery signatures are also different from those of typical events. Given the logarithmic amplitude scale in Figure 2, typical early events are seen to exhibit a recovery time which is initially rapid, slowing down towards the end of the event. The LOREs, on the other hand have a nearly exponential recovery time, which differentiates them from typical early events and indicates that a different set of chemical reactions are responsible for the two recovery signatures. Such a longlasting relaxation process could be attributed to the electron density loss rate, which at upper $D$-region heights is affected mainly by electron attachment and dissociative recombination, and less by electron detachment processes which are much slower, that is, lasting several tens of minutes [e.g., 
VLF amplitude recordings. 2009. Dec. 12, 23:30 - Dec. 13, 00:20 UT

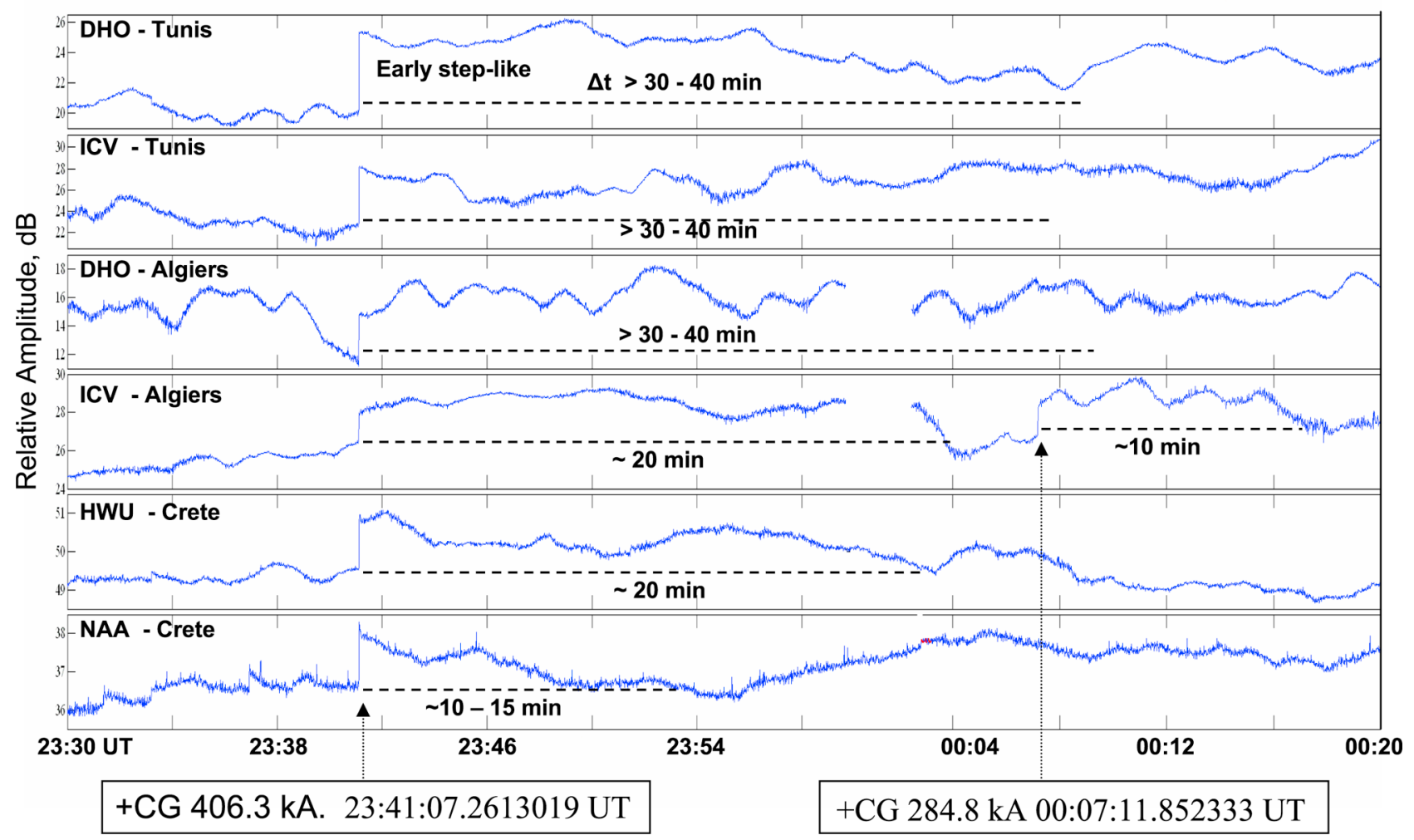

Figure 3. Step-like early VLF events corresponding to the elves shown in Figure 2, both triggered by very intense $+\mathrm{CG}$ lightning strokes. They are observed at by transmitter-receiver GCPs passing at relatively large distances from the causative lightning flashes. These type of early perturbations offset the transmission signal levels for long times. See text for more details.

Glukhov et al., 1992; Rodger et al., 1998; Haldoupis et al., 2009].

[13] The large LORE discussed in Figure 2 was also accompanied by considerable perturbations in VLF signals received in both Algiers and Crete, despite the fact that their GCPs passed more than $250 \mathrm{~km}$ away from the storm center. Interestingly, most of these relatively "far-storm" early signal perturbations were "step-like", a type identified and reported also by Cotts and Inan [2007]. Figure 3 illustrates several such step-like signatures observed at far-storm VLF signal transmissions in Tunis, Algiers and Crete, all of which are coincident with the $+\mathrm{CG} 406.3 \mathrm{kA}$ lightning discharge that caused the massive elve and sprite shown in Figure 2. As seen, step-like perturbations are large (up to $\sim 5 \mathrm{~dB}$ ) and offset the signal level in some cases more than $40 \mathrm{~min}$. In Figure 3, the latter is particularly true for the DHO-Tunis, DHO-Algiers and ICV-Algiers VLF links.

[14] The step-like perturbations described above have not been observed with typical, QE-related early VLF events, apparently because they are signatures associated with a large and spatially extended region of ionization in relation with an elve, which affects not only the lower $E$-region but also the upper $D$-region, down to VLF reflection heights. For example, one could interpret the TLE in Figure 3 that is caused by the +CG 284.8 kA stroke at 00:07:11.8 UT to be elve-dominated. Given the geometries in Figure 1, a possibility exists that the step-like events are due to strong VLF reflections off the boundaries of long-lasting, large horizontally-extended volumes of electron density enhancements in the upper
$D$-region, caused by impacting ionization effects of a lightning-induced EMP field that also excites an elve.

[15] The contribution of a sprite and elve produced by the same $+C G$ discharge is possibly twofold. First, the sprite process and its associated $\mathrm{QE}$ fields can enhance the ionization at VLF reflection altitudes through secondary impact ionization of electrons produced there earlier by the same EMP that also excites the elve. Second, a sprite can contribute to ionization enhancement through a process that involves the effects of sprite halos. Although possibly subvisible, halos could be present in the cases reported in this study. Halos, which appear near the VLF reflection heights to lower altitudes (say, between 90 and $75 \mathrm{~km}$ ), could have a significant effect on ambient electron conductivity because they are caused by more impulsive charge moment changes as compared to typical sprites not accompanied by halos [Qin et al., 2011].

[16] To reinforce these findings, Figure 4 presents VLF and TLE observations for 3 additional cases of LOREs. The figure includes three TLE images which show sprite and elve pairs associated with LORE onsets in different Tx-Rx VLF links, both for near-storm (Tunis) and far-storm (Crete and Algiers) links. These events are less pronounced than the strong case discussed in Figure 2, but they show similar characteristics. Although the causative $+\mathrm{CG}$ lightning discharge went unrecorded by LINET for the middle sprite-elve pair image, there is little doubt it has occurred because high resolution VLF records revealed a powerful sferic to be present at onset in the signals of the links shown in Figure 1. 
VLF recordings (amplitude, dB). Dec. 13. 2009, 01:15 - 02:10 UT

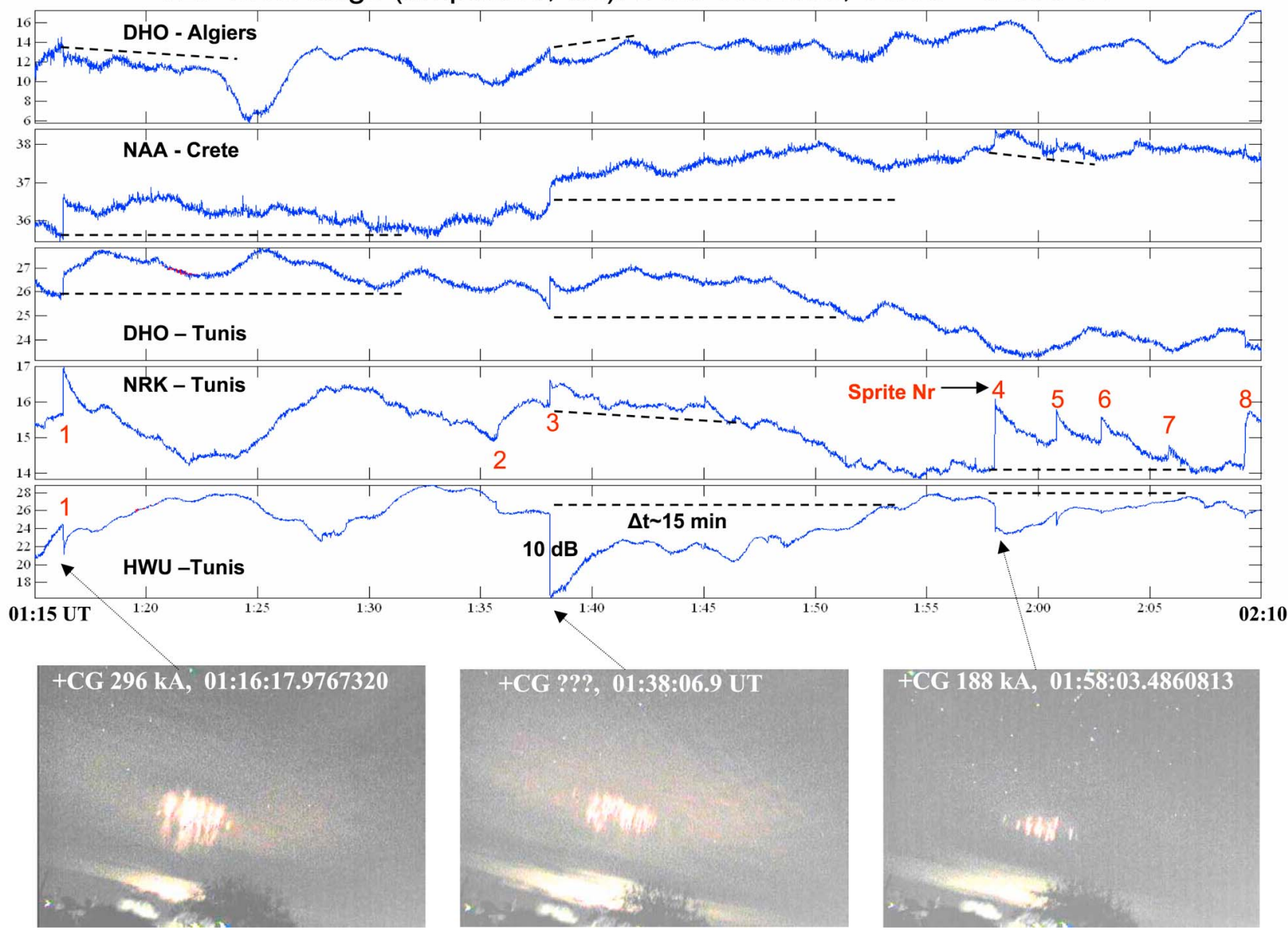

Figure 4. Examples of more LOREs caused by intense + CG discharges and in association with the generation of both, sprites and elves in the upper $D$-region ionosphere.

The right hand-side image that is caused by a + CG $188 \mathrm{kA}$ stroke, also included a sprite-elve pair, but the elve was very faint in this case. Of the 10 LOREs identified in the current analysis 8 were associated with sprite-elve pairs while 2 were associated with $+\mathrm{CG}$ lightning flashes causing sprites only. For the 2 cases where an elve was not observed, it is possible that it may simply have been missed by the relatively slow frame rate of the CCD camera, combined with the very short duration $(\sim 1 \mu \mathrm{s})$ of the elves themselves.

\section{Discussion}

[17] The findings indicate that LOREs, reported first by Cotts and Inan [2007], may occur in relation with lightning-induced electronic conductivity modifications in the upper $D$-region ionosphere rather than heavy ion conductivity changes in the lower atmosphere below $\sim 50 \mathrm{~km}$. Our interpretation of observations suggests that LOREs are likely due to horizontally extended and long-lasting electron density perturbations, at $85 \mathrm{~km}$ or higher, generated by intense $+C G$ lightning discharges that cause large elves followed also by high altitude column sprites. In addition, we suggest that the long enduring step-like early VLF events, also reported first by Cotts and Inan [2007], are due to oblique VLF reflections off the lateral boundaries of large regions of long-lasting elevations in electron density.
This is a postulation and, based on this data alone, one may not exclude the case of narrow-angle forward scattering. This can be because the elve-associated ionization regions are widely extended horizontally outwards and thus are able to perturb GCPs which are at distances larger than those observed with smaller disturbances that produce typical early events ( $<100 \mathrm{~km}$ [e.g., see Johnson et al., 1999]).

[18] The observation showing elves to be nearly always present at the onset of LORE and step-like event occurrences, suggests that the EMP emitted by a strong $+\mathrm{CG}$ lightning discharge plays a primary role in triggering the LORE process. This is likely to be reinforced by the QE effects that follow in time to produce the accompanying high-altitude sprite and possibly sub-visual halos. The present observations indicate, but cannot prove, that the elve-sprite combination is of key importance in the LORE generation mechanism. This is not unlikely, given that typical early events with short recoveries (i.e., $<100$ s) are occurring mostly in relation only with sprites, sprite halos and rarely with elves, because in most cases elves occur at higher altitudes where ionospheric recovery times are intrinsically longer (several minutes to a few tens of minutes [e.g., see Rodger et al., 1998]), but these altitudes are usually above the VLF reflection height. There may thus be a possibility of a coupling mechanism between the sprite and elve that allows the electron density left behind by the elve, 
which is ordinarily too high in altitude in order to affect VLF propagation, to scatter VLF waves when a sprite initiates just below it. On the other hand, one may not exclude that LOREs could occasionally be seen to occur in association only with elves (for example, those produced by very intense - CG discharges), or only with high altitude sprites, and/or sprite halos, which can relate with regions of long lasting electron density enhancements there. In the present data set, this scenario occurred in two cases when LOREs of smaller duration ( $\sim 5$ to $8 \mathrm{~min}$ ) were caused by $+\mathrm{CG}$ lightning of $\sim 180 \mathrm{kA}$ and $\sim 200 \mathrm{kA}$ peak intensities that also caused sprites, but not elves.

[19] The present observations confirm previous results about the effects in the upper $D$-region ambient plasma triggered by strong lightning strokes which are causative of both elves and sprites. Fukunishi et al. [1996], in the first paper on elves, referred to unpublished results mentioning that: "elves were always accompanied by large amplitude VLF perturbations". This motivated them to introduce "elves" for Emissions of Light and $V L F$ perturbations due to EMP Sources, an acronym which was later abandoned, though the term elves persists. In addition, the same authors reported that: "strong elves appear to occur in response to some especially energetic +CG flashes which were accompanied nearly always with sprites". Although it was mentioned, examples of such large and long lasting early VLF perturbations were never reported openly before to occur in relation with elves and sprite pairs. Furthermore, Fukunishi et al. [1996] stated that, "the large horizontal extent and high luminosity of these events suggested a significant effect on the lower ionosphere and on radio wave propagation", which was in line also with theoretical predictions by Taranenko et al. [1993] that appeared prior the elves' discovery. The present observations confirm these earlier predictions and postulations.

[20] In discussing the topic of VLF perturbations associated with elves in his review paper, Rodger [2003] pointed out that "an intense lightning EMP that causes an elve also leads to changes in ionization which at elve-altitudes will be relatively long lasting". He went on to suggest that "the relaxation of such perturbations to pre-event levels would be expected to be extremely slow due to the long lifetimes of electrons at elvesaltitudes, and VLF events would likely appear as sudden steplike changes in received signal amplitude and phase without a clear relaxation signature". These predictions, which are made several years ago by Rodger [2003], are now confirmed for first time by the present LORE and step-like early VLF observations, although they do not seem to match the predicted narrow scattering pattern.

\section{Conclusions}

[21] Out of the 10 LOREs identified in this study 8 were associated with sprite-elve pairs. The results constitute evidence that strong $+\mathrm{CG}$ lightning can cause largely-extended and long-lasting severe modifications of the ionosphere at upper $D$-/lower $E$ - region, which could have significant effects on radio signal propagation. An improved understanding of this phenomenon, however, requires more research and additional data in order to be better quantified. Such work includes theoretical development of an appropriate ionospheric disturbance model as well as theoretical modeling of VLF scattering from such disturbance regions.
In addition, application of an upper $D$-region chemical model may also be needed to simulate the long recoveries of the observed perturbations in plasma density. The application of a simplified model by Rodger et al. [1998] for the upper $D$-region, in which the electron continuity equation includes only dissociative recombination and electron attachment as loss terms, showed that the observed LORE durations are easily accountable. For example, if the nighttime ambient electron density was elevated by 5 to 20 times at $85 \mathrm{~km}$, then it relaxes back to its pre-event levels in about 20 to $25 \mathrm{~min}$, which compares well with the observations. More detailed modeling studies of the present observations will be undertaken in a future study.

[22] Finally we touch upon on a couple of points in brief. One, which was not commented here but could be of importance and needs to be considered more experimentally, is that these exceptional observations associate with a localized maritime thunderstorm, as it was also the case for the large elves observed by Fukunishi et al. [1996] and the great majority of the LORE events reported by Cotts and Inan [2007]. Another point, which was introduced above but certainly needs more consideration and attention, as it may involve important physics behind it, is that LOREs seem to occur overwhelmingly in correspondence to an "elve and sprite" pair, rather than an elve or sprite alone. This is an important indication that would imply the presence of a coupling process between the two phenomena which are driven by different physical processes, that is, EMP and QE fields, acting upon the medium in time sequence. We cannot offer here details of how the mechanism of sprite-elve coupling might work, but do note that existing theories of both sprite and elve production, treated separately, are unable to produce LOREs, the former because the ionization is too low in altitude, and the latter because the ionization usually is too high for VLF scattering to occur. Given our suggestion that LOREs are mostly associated with sprite-elve pairs, which also agrees with the first elves observations of Fukunishi et al. [1996], it is possible that LORE production requires both to be present.

[23] Acknowledgments. We are grateful to colleagues and students at Stanford who keep the international AWESOME VLF network project going strong. Many thanks go to Nino Amvrosiadi and Yannis Tsabos in Crete, as well as to our colleagues Hassen Ghallila in Tunis and Samir Nait Amor in Algiers hosting and maintaining the VLF receiver stations. The lightning data were kindly provided by Stefano Dietrich of the Italian LINET team. Our very special thanks go to Ferruccio Zanotti, an amateur photographer who did a great job in capturing the revealing TLE events used in this study. Partial support for this work was provided by ELKE, University of Crete. Partial support for this paper was provided by ELKE, University of Crete, and the DARPA grant HR0011-10-1-0058-P00001 to Stanford University.

[24] The Editor thanks Robert Moore and an anonymous reviewer for assisting in the evaluation of this paper.

\section{References}

Barrington-Leigh, C. P., U. S. Inan, and M. Stanley (2001), Identification of sprites and elves with intensified video and broadband array photometry, J. Geophys. Res., 106, 1741, doi:10.1029/2000JA000073.

Cohen, M. B., U. S. Inan, and E. W. Paschal (2010), Sensitive broadband ELF/VLF radio reception with the AWESOME instrument, IEEE Trans. Geosci. Remote Sens., 48(1), 3-17, doi:10.1109/TGRS.2009.2028334.

Cotts, B. R. T., and U. S. Inan (2007), VLF observation of long ionospheric recovery events, Geophys. Res. Lett., 34, L14809, doi:10.1029/ 2007GL030094.

Cummer, S. A., and W. A. Lyons (2005), Implications of lightning charge moment changes for sprite initiation, J. Geophys. Res., 110, A04304, doi:10.1029/2004JA010812. 
Fukunishi, H., Y. Takahasi, M. Kubota, K. Sakanoi, U. S. Inan, and W. A. Lyons (1996), Elves: Lightning-induced transient luminous events in the lower ionosphere, Geophys. Res. Lett., 23, 2157-2160, doi:10.1029/96GL01979.

Glukhov, V., V. Pasko, and U. Inan (1992), Relaxation of transient lower ionospheric disturbances caused by lightning-whistler-induced electron precipitation, J. Geophys. Res., 97, 16,971-16,979, doi:10.1029/ 92JA01596.

Haldoupis, C., T. Neubert, U. S. Inan, A. Mika, T. H. Allin, and R. A. Marshall (2004), Subionospheric early VLF signal perturbations observed in one-to-one association with sprites, J. Geophys. Res., 109, A10303, doi:10.1029/2004JA010651.

Haldoupis, C., A. Mika, and S. Shalimov (2009), Modeling the relaxation of early VLF perturbations associated with transient luminous events, J. Geophys. Res., 114, A00E04, doi:10.1029/2009JA014313.

Haldoupis, C., N. Amvrosiadi, B. R. T. Cotts, O. A. der Velde, O. Chanrion, and T. Neubert (2010), More evidence for a one-to-one correlation between sprites and early VLF perturbations, J. Geophys. Res., 115, A07304, doi:10.1029/2009JA015165.

Inan, U. S., S. A. Cummer, and R. A. Marshall (2010), A survey of ELF/ VLF research of lightning-ionosphere interactions and causative discharges, J. Geophys. Res., 115, A00E36, doi:10.1029/2009JA014775.

Johnson, M. P., U. S. Inan, and S. J. Lev-Tov (1999), Scattering pattern of lightning-induced ionospheric disturbances associated with early/ fast VLF events, Geophys. Res. Lett., 26, 2363-2366, doi:10.1029/ 1999GL900521.

Lehtinen, N. G., and U. S. Inan (2007), Possible persistent ionization caused by giant blue jets, Geophys. Res. Lett., 34, L08804, doi:10.1029/ 2006GL029051.

Marshall, R. A., U. S. Inan, and W. A. Lyons (2006), On the association of early/fast very low frequency perturbations with sprites and rare examples of VLF backscatter, J. Geophys. Res., 111, D19108, doi:10.1029/ 2006JD007219.

Mika, Á., and C. Haldoupis (2008), VLF studies during TLE observations in Europe: A summary of new findings, Space Sci. Rev., 137, 489-510, doi:10.1007/s11214-008-9382-8.

Moore, C. R., C. P. Barrington-Leigh, U. S. Inan, and T. F. Bell (2003), Early/fast VLF events produced by electron density changes associated with sprite halos, J. Geophys. Res., 108(A10), 1363, doi:10.1029/2002JA009816.

Pasko, V. P., U. S. Inan, Y. N. Taranenko, and T. F. Bell (1995), Heating, ionization and upward discharges in the mesosphere due to intense quasi-electrostatic thundercloud fields, Geophys. Res. Lett., 22, 365, doi:10.1029/95GL00008.

Pasko, V. P., M. A. Stanley, J. D. Mathews, U. S. Inan, and T. G. Wood (2002), Electrical discharge from a thundercloud top to the lower ionosphere, Nature, 416, 152-154, doi:10.1038/416152a.

Poulsen, W. L., T. F. Bell, and U. S. Inan (1993), The scattering of VLF waves by localized ionospheric disturbances produced by lightninginduced electron precipitation, J. Geophys. Res., 98, 15,553-15,559, doi:10.1029/93JA01201.

Qin, J., S. Celestin, and V. P. Pasko (2011), On the inception of streamers from sprite halo events produced by lightning discharges with positive and negative polarity, J. Geophys. Res., 116, A06305, doi:10.1029/ 2010JA016366.

Rodger, C. J. (2003), Subionospheric VLF perturbations associated with lightning discharges, J. Atmos. Sol. Terr. Phys., 65, 591-606, doi:10.1016/S1364-6826(02)00325-5.

Rodger, C. J., O. A. Molchanov, and N. R. Thomson (1998), Relaxation of transient ionization in the lower ionosphere, J. Geophys. Res., 103, 6969-6975, doi:10.1029/98JA00016.

Scherrer, D., M. B. Cohen, T. Hoekesma, U. S. Inan, R. Mitchell, and P. Scherrer (2008), Distributing space weather monitoring instruments and educational materials for IHY 2007: The AWESOME and SID project, Adv. Space Res., 42, 1777-1785, doi:10.1016/j.asr.2007.12.013.

Taranenko, Y. N., U. S. Inan, and T. F. Bell (1993), The interaction with the lower ionosphere of electromagnetic pulses from lightning: Excitation of optical emissions (1993), Geophys. Res. Lett., 20, 2675-2678, doi:10.1029/93GL02838.

van der Velde, O. A., J. Bor, J. Li, S. A. Cummer, E. Armone, F. Zannotti, M. Fullekrug, C. Haldoupis, S. Nait-Amor, and T. Farges (2010), Multiinstrument observations of a positive gigantic jet produced by a winter thunderstorm in Europe, J. Geophys. Res., 115, D24301, doi:10.1029/ 2010JD014442. 